\title{
Composite electrospun nanomembranes of fish scale collagen peptides/chito-oligosaccharides: antibacterial properties and potential for wound dressing
}

\author{
This article was published in the following Dove Press journal: \\ International Journal of Nanomedicine \\ | April 201 I \\ Number of times this article has been viewed
}

Yan Wang'

Chen-lu Zhang ${ }^{2}$

Qun Zhang'

Ping $\mathrm{Li}^{1,3}$

'School of Life Sciences and Technology, Tongji University, Shanghai, People's Republic of China; ${ }^{2}$ College of Life Sciences, Nanjing Agricultural University, Nanjing, People's Republic of China; ${ }^{3}$ Shanghai Key Laboratory of Molecular Catalysis and Innovative Materials, Fudan University, Shanghai, People's Republic of China
Correspondence: Ping Li School of Life Sciences and Technology, Tongji University, 1239 Siping Road, Shanghai 200092,

People's Republic of China

Tel +862 I 6598 I05I

Fax +862165988653

Email liping0I@tongji.edu.cn
Purpose: The objective of the present investigation was to evaluate the antibacterial properties and the biocompatibility of composite electrospun nanofibrous membranes (NFMs) with low-molecular-weight fish scale collagen peptides (FSCP) and chito-oligosaccharide (COS), to determine their potential for use as wound dressings.

Methods: Low-molecular-weight FSCP were combined with COS to prepare nanofibers by electrospinning, and polyvinyl alcohol (PVA) was used for enhancing fiber-forming ability. Transmission electron microscope and scanning electron microscope methods were used to observe bacterial adhesion and the bacterial cell membrane. Fibroblast cell viability was determined using the 3-(4,5-dimethylthiazol-2-yl)-2,5-diphenyltetrazolium bromide (MTT) assay.

Results: The best FSCP/COS mass ratio for electrospinning was 2:1, and the nanofibers had small dimensions ranging from 50 to $100 \mathrm{~nm}$. The NFM showed good antibacterial activities against Gram-positive Staphylococcus aureus and Gram-negative Escherichia coli. The antibacterial activity against $S$. aureus was higher than against $E$. coli. The pili and adhesive fimbriae of $E$. coli promoted bacterial adhesion to the NFM surfaces, and $S$. aureus biofilms aided $S$. aureus adhesion on the surface of NFMs. Damage to the bacterial cell membrane indicates that the NFMs could lead to the release of intracellular materials, particularly with $S$. aureus. In addition, FSCP/COS NFM rapidly increased the permeability of the outer membranes of E. coli. The electrospun NFM with FSCP and COS had good biocompatibility in vitro and supported proliferation of human skin fibroblasts.

Conclusion: FSCP are superior to mammalian collagen, and have feasibility and potency for wound dressings. FSCP/COS NFMs had good anti-bactericidal activity that improved with increased COS, and showed good biocompatibility in vitro and supported the proliferation of fibroblasts.

Keywords: composite electrospun nanomembranes, fish scale collagen peptides, bacterial adhesion, bacterial cell membrane, wound dressing

\section{Introduction}

Nanomaterials are a new type of biomedical material that may have a wide range of advantages as medical dressings, including strong adsorption and the ability to assume variable shapes. They can act as antimicrobial barrier films to prevent wound infection and bleeding, and to promote wound healing. ${ }^{1}$ Electrospinning (ES) is a simple and effective nanofabrication method for preparing nanofibers, with diameters ranging from 5 to $500 \mathrm{~nm}, 10^{2}$ to $10^{4}$ times smaller than those 
prepared by traditional methods. ${ }^{2}$ Because of the very high surface area, together with many small secondary structures, electrospun NFMs have a strong adhesive force, good air filtration, high adhesion barrier activity, and heat resistance. ${ }^{3-6}$ Their structures make NFMs similar to the skin structure of the human body. Some electrospun membranes have been used specifically in biomedical fields for purposes such as biofilms, wound dressing materials, hemostatic materials, artificial blood vessels, drug and gene delivery, and tissue engineering scaffold materials. ${ }^{2,7}$ High-molecular-weight (MW) polymers are good materials for nanofiber preparation, but to date, the feasibility of electrospinning low-MW polymers such as collagen peptides with MW less than 10,000, combined with COS, has not been studied.

Most collagens used for nanomaterials are from pig and cattle skin and bones, or mammalian leathers. ${ }^{8,9}$ In recent years the occurrence of human-animal cross-infections has increased concern about using collagen extracts from terrestrial animal sources. ${ }^{10}$ Consequently, extracts from fish skins, scales, and bones have become a potential alternative source for collagen, and may be superior to mammalian collagen because of the absence of the risk of animalrelated contamination. However, there have been no reports concerning the feasibility of using fish scale collagen for nanomaterials preparation. Collagen peptides (CP) are low-MW fragments from collagen. ${ }^{11}$ Compared with collagen, which has a MW of 300,000 Da and above, collagen peptides enable easier direct absorption by the human body. The lower-MW collagen peptides not only go through the skin's stratum corneum, but also penetrate the deep dermal layer. Furthermore, they have the same important role as human peptides in tissue repair and reconstruction of skin. ${ }^{12}$ Collagen peptides can extend the life of skin cells, inhibit lipid peroxidation, and defend against oxidative free radical attacks on DNA. ${ }^{13}$ In addition, they have other effects such as: the inhibition of tyrosinase activity; improvement of skin immunity and skin water-holding capacity; and promotion of collagen synthesis and dermal hyaluronic acid synthesis. FSCP of low MW have low antigenicity and are nonallergenic, but no work has been done on using them in wound dressings.

Chitosan has a wide inhibition spectrum for Gram-positive and Gram-negative bacteria, yeasts, and molds. Also, it has no cytotoxicity, causes no hemolysis, contains no known allergens, and has excellent biocompatibility and good biological safety, so chitosans from various sources are widely used in the fields of biomedical materials, textiles, and food additives. The oligomer of chitosan, COS, is water-soluble and has similar biological activity to chitosan. With respect to wound dressing, because of the relatively rapid interaction between the wound and the water-soluble, easily swelling COS in a moist healing environment, $\mathrm{COS}$ is a more effective woundhealing accelerator than chitosan. PVA is a hydrophilic, nontoxic, biocompatible, and fully degradable synthetic polymer, with excellent film-forming, emulsifying, and adhesive properties. ${ }^{14}$ Therefore, materials blended with PVA have a great affinity for skin and extracellular matrix materials. Only a few papers have reported on the preparation of electrospun nanomembranes of collagen or chitosan for wound dressing. ${ }^{15-17}$ Moreover, to date no work has been reported on the electrospinning of low-MW FSCP and COS, and whether or not they could be combined with PVA to obtain composite nanofibrous membranes for wound dressing. Peptide- or collagen peptide-based wound dressing products are still in the early stages of research. Recently, a few studies have focused on the use of peptides for early-stage wound healing. ${ }^{18}$

In this study, low-MW FSCP and COS were electrospun to form composite nanofibers. The antibacterial properties of the composite nanofibrous membranes were then evaluated, using two typical clinical wound pathogens: Staphylococcus aureus and Escherichia coli. The potency of the composite nanomembranes for wound dressing was tested using the 3-(4,5-dimethylthiazol-2-yl)-2,5-diphenyltetrazolium bromide (MTT) method with clinically relevant human skin fibroblasts.

\section{Materials and methods Materials}

For electrospinning the fish scale collagen peptide/COS nanofibrous membranes, FSCP with an average manufacturer's specified MW of 800 Da were used (Hubei Reco Bio-Tech Co Ltd, Wuhan, China), and chitosan, with a manufacturer's specified MW of $8 \mathrm{kDa}$ and deacetylation degree of $90 \%$, was purchased from Shanghai Lanji Technology Development Co, Ltd (Shanghai, China). PVA was purchased from Sinopharm Chemical Reagent Co, Ltd (Shanghai, China). The cells used in this study were human skin fibroblasts (HSF) (Anhui Medical University, Hefei, China). Fetal bovine serum (FBS), dimethyl sulfoxide (DMSO), and Dulbecco's modified Eagle's medium (DMEM) were purchased from Sigma-Aldrich Chemical Co (St Louis, MO). All other chemicals were analytical grade. 


\section{Types of bacteria}

The strains used for antimicrobial activity measurements were S. aureus ATCC 6538 and E. coli ATCC 8739.

\section{Preparation of FSCP/COS nanofibrous membrane}

A series of FSCP, COS, and PVA solutions was prepared by dissolving collagen peptides and PVA in water, and COS in methanoic acid, respectively. After PVA was dissolved with at a contentration of $6 \%$, and the contentrations of FSCP and $\mathrm{COS}$ were $12 \%$. The $\mathrm{FSCP} / \mathrm{COS}$ mass ratios were varied between 1:3, 1:2, 1:1, 2:1, and 3:1. During electrospinning, nanofibers were collected on aluminum foil by connecting a high voltage power supply (GDW-A, Beijing Institute of High Voltage Electrical and Mechanical Technology Inc, Beijing, China). A syringe pump (WZ-50C2, Zhejiang University Medical Instrument Co Ltd, Hanzhou, China) was used to deliver the polymer solution at flow rates from 0.3 to $1.5 \mathrm{~mL} /$ hour. Electrospinning was done at voltages from 20 to $50 \mathrm{kV}$ with a needle to a collector gap distance of 5 to $15 \mathrm{~cm}$. Finally, the treated membrane was dried for 7 days in a vacuum oven at $37^{\circ} \mathrm{C}$.

\section{Nanofiber characterization}

The morphology of electrospun FSCP/COS complex fibers was observed using scanning electron microscopy (SEM) (XL-30E; Philips, Tokyo, Japan) operating with an accelerating voltage of 10 or $15 \mathrm{kV}$. Prior to SEM, the samples were sputter coated with gold. Based on the SEM micrographs, the average diameter and diameter distribution were determined by choosing 100 fibers at random from $1000 \times$ magnification SEM images and analyzing them using image analysis software (Adobe Photoshop 7.0; Adobe Systems Inc, San Jose, CA).

Fourier transform infrared (FTIR) spectroscopy studies were done on compressed films containing potassium bromide pellets and sampled using an FTIR spectrophotometer (Avatar 380; Thermo Scientific, Austin, TX). All spectra were recorded in absorption mode at $2 \mathrm{~cm}^{-1}$ interval wavenumbers from 600 to $3800 \mathrm{~cm}^{-1}$.

\section{Antimicrobial properties of FSCP/COS nanofibrous membranes}

The different FSCP/PVA, COS/PVA, FSCP/COS/PVA, and PVA NFMs were treated with UV for 20 minutes. Then $1 \mathrm{~mL}$ of the test strain $\left(10^{6} \mathrm{CFU} / \mathrm{mL}\right)$ of $S$. aureus or E. coli was added to the surface of the NFM and incubated at $37^{\circ} \mathrm{C}$ for 10 hours. The residual colonies were washed from the
NFM and then the decrease in colonies on incubated agar plates was used to evaluate antimicrobial activity. The PVA NFM sample served as the control. The bactericidal activity was calculated using the following equation: Antimicrobial activity $(\%)=\{(\mathrm{C}-\mathrm{T}) / \mathrm{C}\} \times 100$, where $\mathrm{C}$ is the number of colonies counted for the control and $\mathrm{T}$ is the number of colonies obtained from each tested sample.

The morphology of the bacterial adhesion was observed by SEM.

\section{Integrity of bacterial cell membrane analyses}

Bacteria were cultured in liquid lysogeny broth medium in tubes at $37^{\circ} \mathrm{C}$. The electrospun membranes were cut into $2 \mathrm{~cm} \times 2 \mathrm{~cm}$ squares in a sterile environment and one placed into each tube. After incubating for different times, bacterial cells were washed and then distributed on copper mesh. Cell morphology was examined by transmission electron microscopy (TEM) (JEM-2010; JEOL, Tokyo, Japan) at Tongji University in China. The release of material from bacteria after treatment with NFMs was examined by determining the absorption values at $260 \mathrm{~nm}$ and $280 \mathrm{~nm} \cdot{ }^{19}$ Bacterial cultures were harvested, washed and resuspended in sterile physiological saline. The final cell suspension was adjusted to an absorbance at $420 \mathrm{~nm}$ of 0.8 . A $2 \mathrm{~cm} \times 2 \mathrm{~cm}$ portion of membranes was mixed with $3.0 \mathrm{~mL}$ of each bacterial cell suspension. PVA NFMs served as the control, and the release over time of materials absorbing at $260 \mathrm{~nm}$ and $280 \mathrm{~nm}$ was monitored with a 1601 UV-VIS spectrophotometer (Shimadzu, Tokyo, Japan).

\section{Fibroblast cell viability tests}

Human skin fibroblasts were seeded in 6-cm culture dishes and cultured in DMEM. The samples for fibroblast cell viability tests were FSCP, COS, PVA, FSCP/COS NFM, and PVA as a positive control. The NFMs were cut into $2 \mathrm{~cm} \times 2 \mathrm{~cm}$ squares and placed into the center of the dish. After incubating for different times, cell morphology was examined using an inverted optical microscope (IX-81; Olympus, Tokyo, Japan).

MTT methods were based on the method of Natthan et al with modification. ${ }^{20}$ The different electrospun membranes were sterilized by UV radiation for 1 hour, then immersed in a serum-free medium containing only DMEM, in an incubator for 24 hours to produce an extraction media of $1 \mathrm{mg} / \mathrm{mL}$. Fresh culture medium (DMEM), was supplemented with $10 \%$ FBS, and used as a negative control. HSF cells were plated in $90 \mu \mathrm{L}$ of DMEM, at a density of $1 \times 10^{4}$ cells $/$ well in 96 -well plates, and cultured at $37^{\circ} \mathrm{C}$ under a $5 \% \mathrm{CO}_{2} / 95 \%$ 

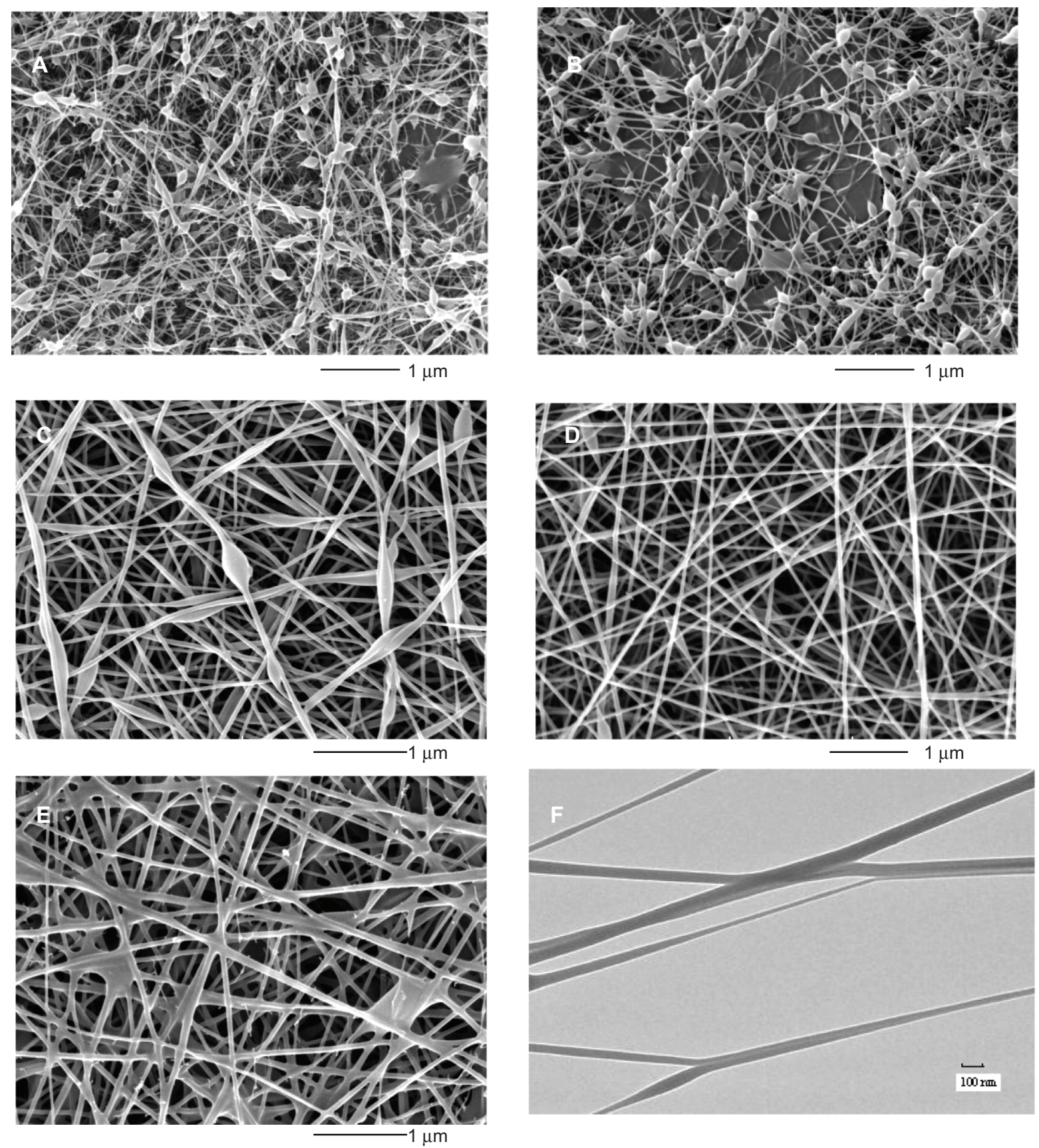

Figure I SEM images of FSCP/COS NFMs with different FSCP/COS mass ratios of I:3 (A), I:2 (B), I:I (C), 2:I (D), and 3:I (E); TEM image of FSCP/COS NFM with FSCP/ COS at a mass ratio of 2:I (F).

Abbreviations: COS, chito-oligosaccharides; FSCP, fish scale collagen peptides; NFMs, nanofibrous membranes.

atmosphere. When the cells were cultured for 24 hours after plating, the tested extraction media were replaced and the cells were reincubated for 24 hours. After treatment for different times, the tested extraction solutions were removed. Finally, the cells were incubated with $100 \mu \mathrm{L}$ of a MTTcontaining medium $(1 \mathrm{mg} / \mathrm{mL})$ for 4 hours. After the medium was removed, the cells were rinsed with phosphate buffered saline ( $\mathrm{pH}$ 7.4), and the formazan crystals formed in living cells were dissolved in $100 \mu \mathrm{L}$ DMSO per well. Relative viability (\%) was calculated based on the absorbance at $570 \mathrm{~nm}$ using a microplate reader (MicroReader 4; Hyperion, Miami, FL).

\section{Statistical analyses}

The data analyses were performed using SPSS (SPSS Inc, Chicago, IL). The values were reported as mean \pm SD for all of the results. The $P$-values were 2 -tailed and $P<0.05$ was considered as significant. 


\section{Results and discussion Electrospun FSCP/COS fibers}

Due to the large net charges of collagen peptide and COS, they cannot form nanofibers during electrospinning. PVA can be used to decrease the conductivity of the solution, so it is well suited to enhancing fiber-forming ability. ${ }^{21}$ For electrospinning of soluble FSCP combined with COS, methanoic acid was used to dissolve COS. The viscosity and conductivity of the solution, the spinning voltage, the flow rate, and the collecting distance were adjusted in preliminary experiments to optimize the electrospinning conditions. During electrospinning of a FSCP/COS solution, the solution viscosity was found to play an important role in nanofiber formation. COS was more viscous than FSCP. When the COS/FSCP mass ratio was more than 1:1, many beads were observed in the nanofibers (Figures 1A and 1B). When the COS/FSCP mass ratio was less than 1:1, most fibers had smooth surfaces with no phase separation, which indicates that FSCP was compatible with COS and PVA (Figures 1C-1F). Some split and ribbon-type fibers were also observed (Figures 1C-1E), which may have resulted from solvent evaporation during the electrospinning process.

Instead of electrospinning collagen from calf skin using 1,1,1,3,3,3-hexafluoroisopropanol as a solvent, ${ }^{8}$ in our study, only one organic solvent, methanoic acid, with relatively lower toxicity, was used for FSCP/ COS electrospinning. The FSCP/COS fibers have smaller dimensions than mammal collagen fibers from calf skin, with diameters ranging from 50 to $100 \mathrm{~nm}$ (Figure 1E). ${ }^{8}$

To evaluate the composite NFM and its constituent polymers, FTIR spectra were used. After mixing with COS and PVA, the IR spectrum of FSCP changed markedly (Figure 2). For the composite NFM, peaks at 3788 and $1093 \mathrm{~cm}^{-1}$ were assigned to the stretching vibration of $-\mathrm{OH}$ and $\mathrm{C}-\mathrm{O}$ in $\mathrm{COS}$. In addition, the peak between 3000 and $3500 \mathrm{~cm}^{-1}$ was assigned to the free $-\mathrm{OH}$, and the wide and strong peak was attributed to the increase in the degree of intermolecular association for hydroxyl groups. An absorption peak at $849 \mathrm{~cm}^{-1}$ was also found in the spectrum of NFM, which was assigned to the $-\mathrm{CH}_{2}$ in PVA. Typical peaks for collagen peptides were clearly observed, such as amide I at $1640 \mathrm{~cm}^{-1}$ and the flexing vibrations of $\mathrm{N}-\mathrm{H}$ at $654 \mathrm{~cm}^{-1}$ (Figure 2D). For the composite NFM, the characteristic peaks of collagen peptide, COS, and PVA were identified, which confirms that the membrane was composed of all three polymers.

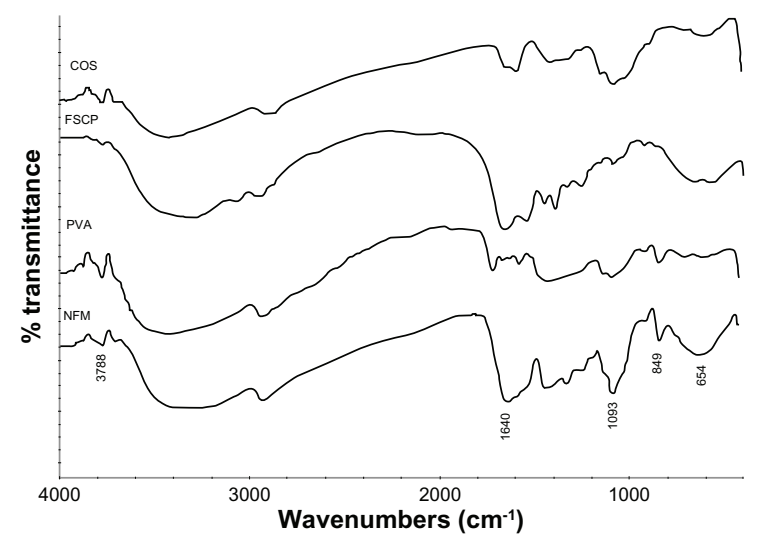

Figure 2 FTIR spectra of COS, FSCP, PVA, and FSCP/COS NFMs.

Abbreviations: COS, chito-oligosaccharides; FSCP, fish scale collagen peptides; NFM, nanofibrous membrane; PVA, polyvinyl alcohol.

\section{Antimicrobial activity of FSCP/COS NFMs}

Antimicrobial activity is considered to be one of the most important properties directly linked to wound dressing. FSCP/COS NFMs showed higher antimicrobial activity against $S$. aureus than against $E$. coli. The half maximal inhibitory concentration $\left(\mathrm{IC}_{50}\right)$ value of $\mathrm{FSCP} / \mathrm{COS} \mathrm{NFM}$ with $S$. aureus was $0.144 \mathrm{~g} / \mathrm{mL}$, lower that for $E$. coli, with an $\mathrm{IC}_{50}$ value of $0.191 \mathrm{~g} / \mathrm{mL}$ (Figure 3A). The time-dependence of antibacterial activities of different NFM-treated bacteria are shown in Figures 3B and 3C. NFM containing COS showed considerable bactericidal activity against E. coli, whereas that without COS was somewhat less active. Therefore, $\mathrm{COS}$ plays an important role in the antibacterial activity of FSCP/COS NFMs.

\section{Bacterial adhesion and integrity of bacterial cell membranes}

In electrospinning, a polymer solution is pushed out of a nozzle towards a surface under an applied voltage, which produces a disordered fibrous surface with uniform features and roughness. It is also possible to control the direction of the fibers on the surface..$^{22}$ An extra advantage is that this type of fibrous surface may mimic the structure of the extracellular matrix. ${ }^{23}$ From electron microscopy photographs, E. coli tightly adhered to the NFMs and $S$. aureus biofilms were formed on the surface of FSCP/COS NFMs (Figure 4). It has been reported that some bacteria may have fimbriae, called pili or curli, which aid in attachment to surfaces. ${ }^{24}$ Many E. coli strains are motile and generally have both sex pili and adhesive fimbriae (Figure 5A1). The pili and adhesive fimbriae of $E$. coli promoted adhesion to the NFM's surfaces (Figure 4). Whereas single cells can adhere to surfaces independently of each other, bacteria 
A

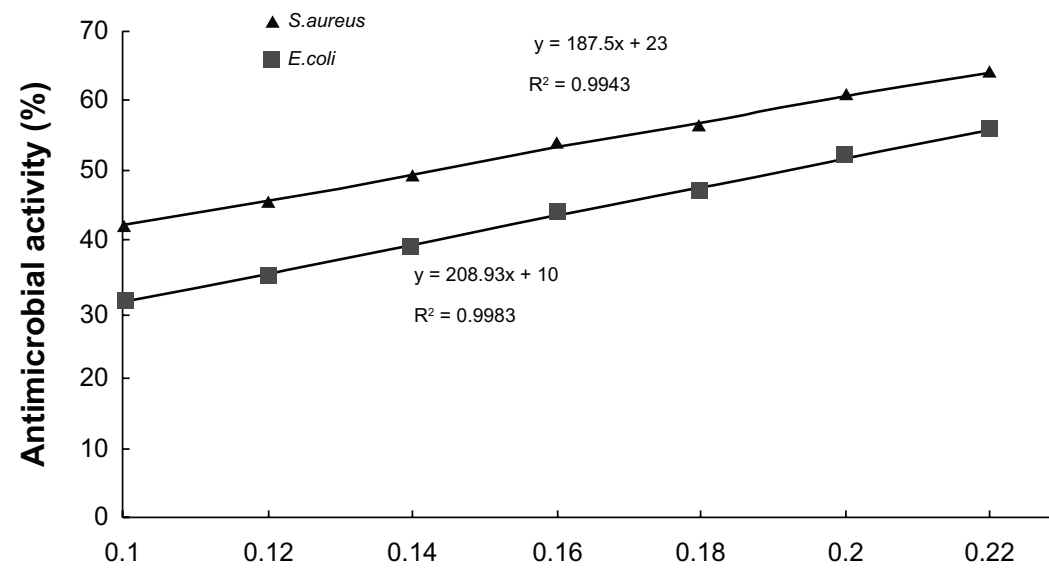

FSCP/COS NFMs (g/mL)

B

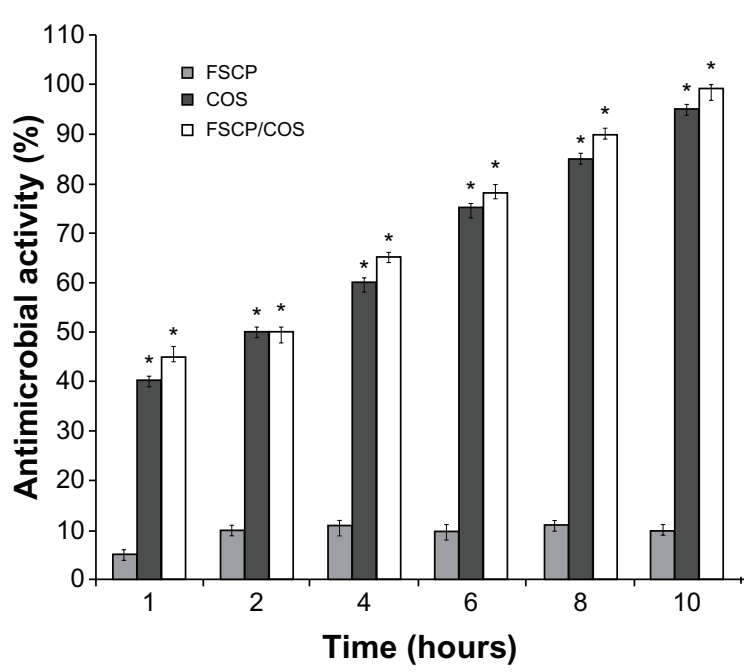

C

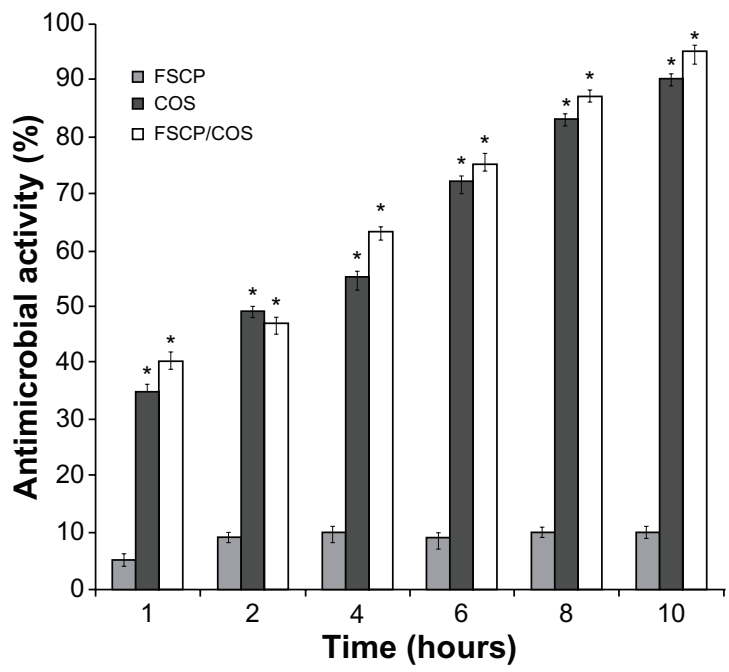

Figure 3 Antibacterial activities of different concentration of FSCP/COS NFMs against S. aureus ( $\mathbf{\Delta})$ and E. coli ( $\mathbf{\square})(\mathbf{A})$; Antibacterial activities of different NFMs at $0.8 \mathrm{~g}$ per sample against $S$. aureus (B), and E. coli (C)

Note: $* P<0.05$ compared with FSCP NFMs.

Abbreviations: COS, chito-oligosaccharides; FSCP, fish scale collagen peptides; NFM, nanofibrous membranes.
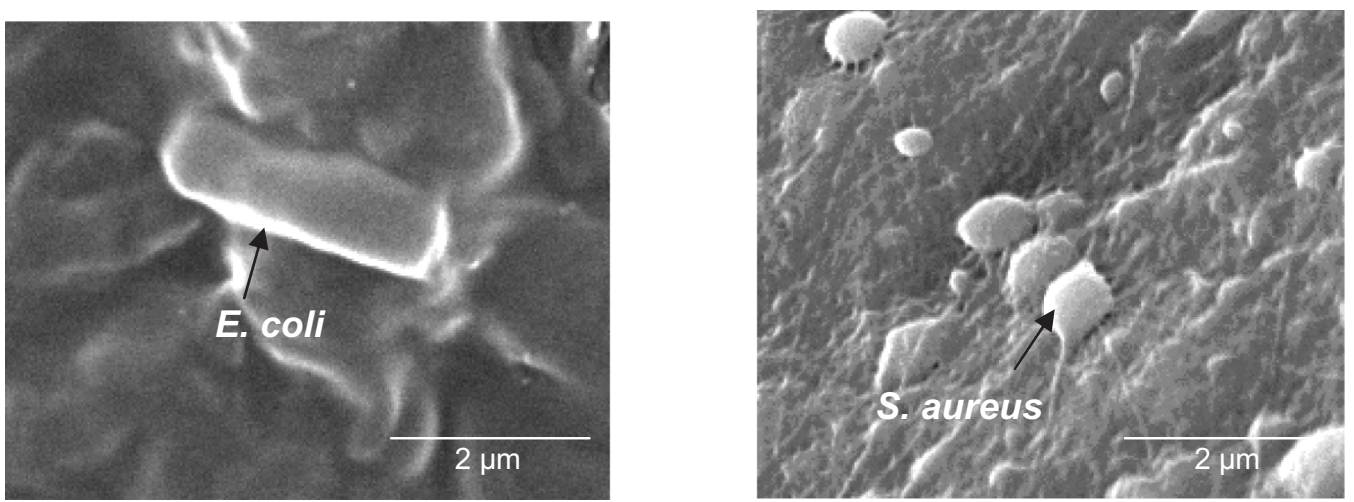

Figure 4 SEM images of bacteria attached on the surfaces of FSCP/COS NFMs after 2 hours of incubation.

Abbreviations: COS, chito-oligosaccharides; FSCP, fish scale collagen peptides; NFMs, nanofibrous membranes. 


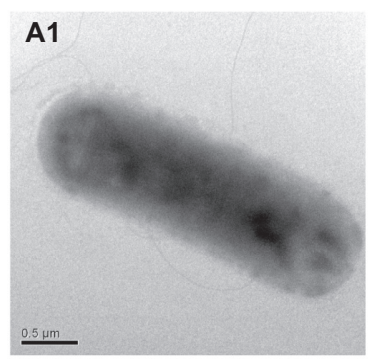

A2
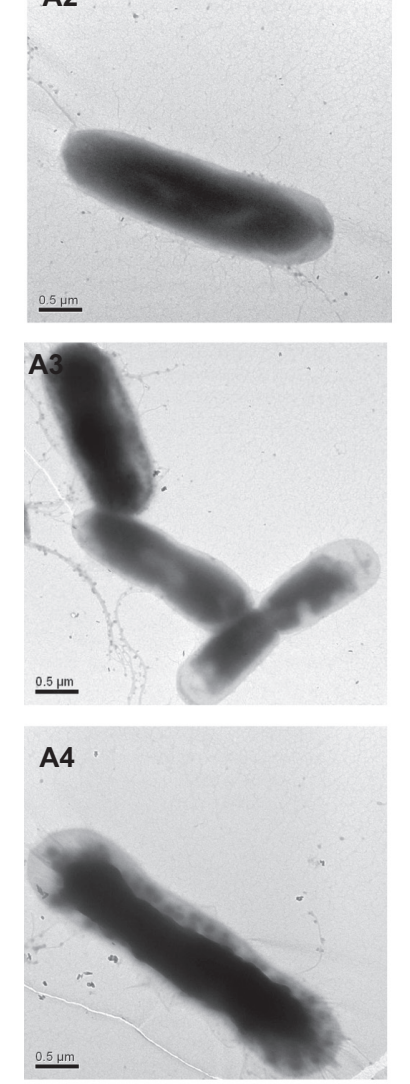

A5

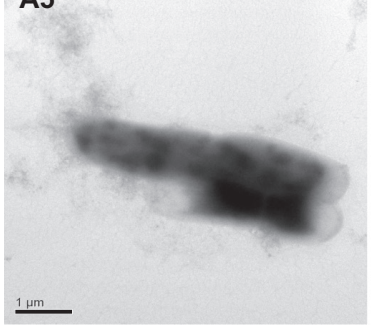

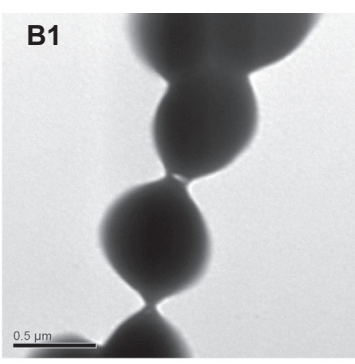

B2
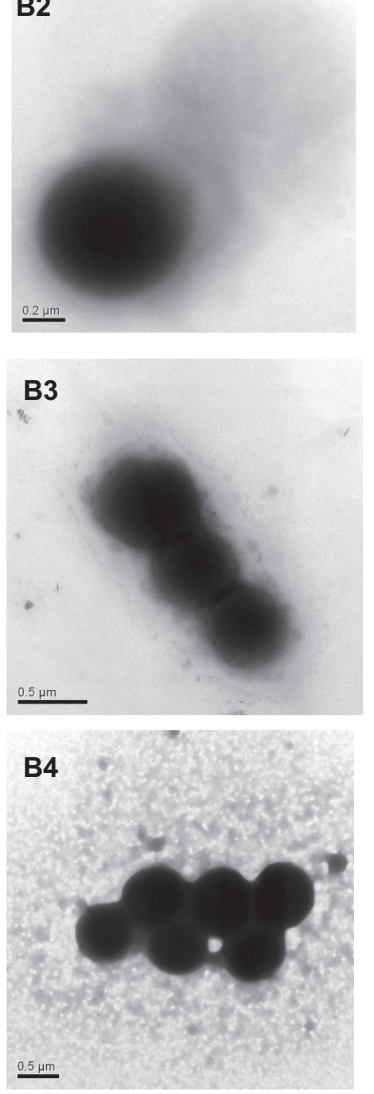

B5

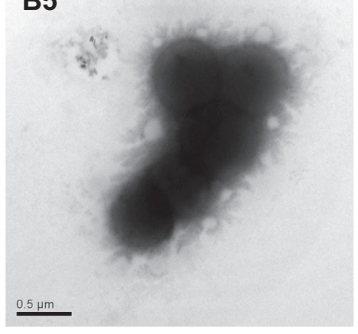

Figure 5 Photomicrographs of $E$. coli (A), and S. aureus (B) treated with FSCP/ COS NFMs. I, 2, 3, 4, and 5 refer to the samples at $0,15,30,45$, and 60 minutes, respectively.

Abbreviations: COS, chito-oligosaccharides; FSCP, fish scale collagen peptides; NFMs, nanofibrous membranes.

live on surfaces as a community, within a species, using strain-specific extracellular polymeric substances (EPS) for binding. This assembly of bacteria and EPS is termed a biofilm. ${ }^{25,26}$ S. aureus is a prominent bacterial pathogen known to form biofilms on many medical implants and host

tissues. ${ }^{27}$ The FSCP/COS NFM permitted the Gram-positive bacterium $S$. aureus to form biofilms on the membrane surfaces. Furthermore, the biofilms strongly influenced S. aureus adhesion on the surface of NFMs (Figure 4).

Integrity of bacterial cell membranes was tested and the results show that FSCP/COS NFM treatment resulted in bacterial cell deformation (Figure 5). Compared with the control, when treated for a prolonged time $E$. coli were disrupted and covered by an additional tooth-like layer (Figure 5A). The photomicrographs show that FSCP/COS NFM damaged the cell membranes of dividing cells. In photomicrographs of FSCP/COS NFM-treated S. aureus, the membranes of cells were disrupted in the constricting region (Figure 5B3-5B5), with loss of cell contents through leakage (Figure 5B2). However, cells of $S$. aureus that were not dividing were not visibly affected except for the thickening of their cell walls. Thus, membrane damage may be one mechanism for inactivation of both E. coli and S. aureus treated with FSCP/ COS NFMs.

The cytoplasm and membranes of bacteria are the target for many inhibitory agents. When bacterial membranes become compromised by interaction with inhibitory agents, initially the low-MW substances such as $\mathrm{K}^{+}$and $\mathrm{PO}^{3-}$ tend to leach out, followed by DNA, RNA, protein and other higher MW materials. The intracellular nucleotides in solution are easily detected by absorption at $260 \mathrm{~nm}$, and the protein at $280 \mathrm{~nm}$, which serves as an indication of membrane damage. ${ }^{19}$ When E. coli suspensions were treated with FSCP/COS NFMs, the $\mathrm{OD}_{260}$ values increased rapidly at first then at a decreasing rate after 60 minutes (Figure 6A). When $S$. aureus suspensions were treated with the NFMs, the $\mathrm{OD}_{260}$ values also increased at first but there was no further increase after 40 minutes. $\mathrm{OD}_{260}$ values were greater for $S$. aureus suspensions than $E$. coli. Thus, the damage to cell membranes for $S$. aureus was greater than for E. coli, which agreed with the findings for bactericidal activity. Jeon et $\mathrm{a}^{28}$ found that when both E. coli and $S$. aureus were treated with dimethylaminoethyl-chitosan, the release of $260 \mathrm{~nm}$-absorbing materials quickly increased, but due to the difference in cell structures, the absorbance values for $E$. coli and $S$. aureus were different. ${ }^{28}$

The release of intracellular proteins from both E. coli and $S$. aureus treated with FSCP/COS NFMs is shown in Figure 6B. When NFM was mixed with the bacterial suspension, the initial absorption at $280 \mathrm{~nm}$ markedly increased. $\mathrm{OD}_{280}$ values increased up to 40 minutes for $S$. aureus, and 60 minutes for $E$. coli, respectively, but little change was found in OD thereafter, suggesting completion of leakage. Composite NFMs containing COS appeared to be more 
A

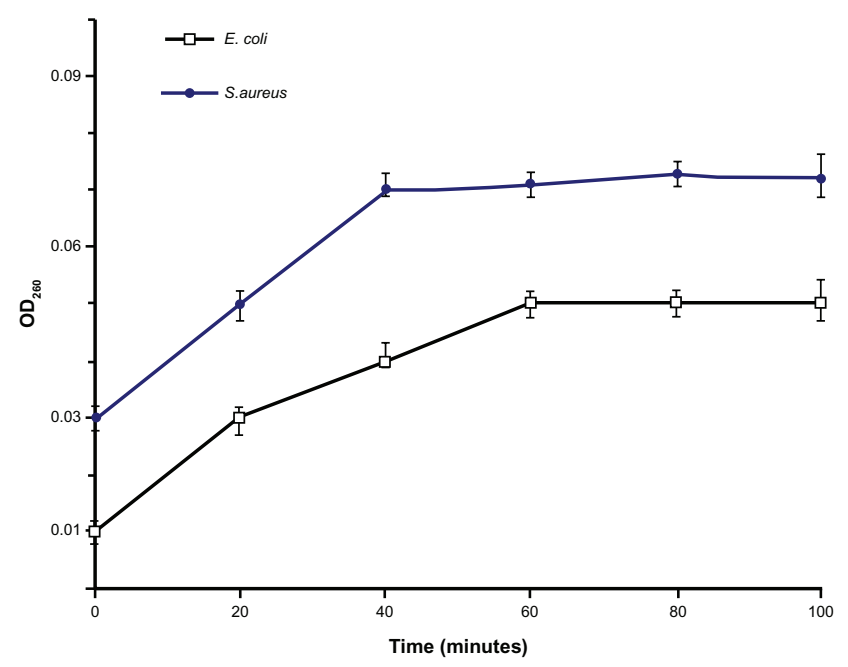

B

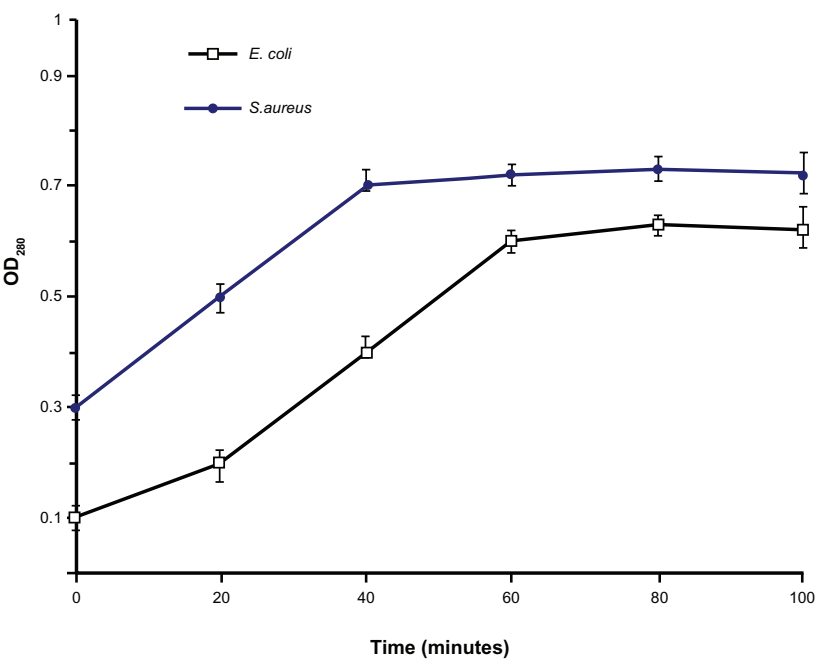

Figure 6 Release of cell materials absorbing at $260 \mathrm{~nm}$ (A) and 280 (B) from E. coli or S. aureus suspensions treated with FSCP/COS NFMs, and PVA NFM treatment as a control.

Abbreviations: COS, chito-oligosaccharides; FSCP, fish scale collagen peptides; NFMs, nanofibrous membranes; PVA, polyvinyl alcohol.

effective for $S$. aureus, which was also consistent with the results of the antimicrobial activity assays.

\section{Fibroblast cell viability}

The morphology of human skin fibroblast cells cultured with various NFMs for 1 and 7 days was examined. After culture for 1 day, the fibroblasts treated with COS, FSCP, and FSCP/ COS NFMs had tended to expand completely, and clear tentacles were found in many fibroblast cells (Figures 7C-7H). However, fibroblasts treated with PVA still kept the long spindle (Figures 7A and B). After 7 days, the number of fibroblasts in cultures treated with PVA NFMs seemed to be fewer than with the other three NFM treatments, and it was observed that FSCP/COS NFMs were very beneficial for the
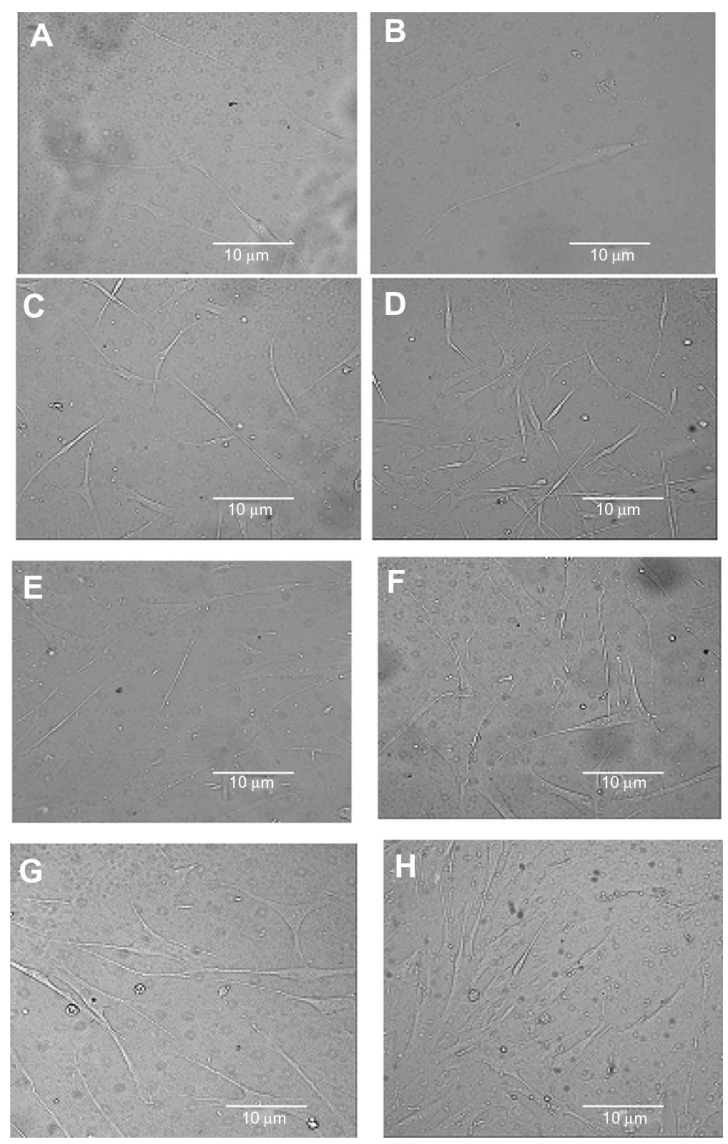

Figure 7 Photomicrographs of human skin fibroblasts after growing for I day and 7 days, respectively, with treatments of PVA (A, B), COS (C, D), FSCP (E, F), and $\operatorname{FSCP} / \mathrm{COS}$ (G, $\mathbf{H})$.

Abbreviations: COS, chito-oligosaccharides; FSCP, fish scale collagen peptides; NFM, nanofibrous membranes; PVA, polyvinyl alcohol.

growth of fibroblasts (Figure 7H). Fibroblast cell proliferation was tested using the MTT methods with different NFMs. MTT absorbance for fibroblast cells grown in culture treated with PVA NFMs was significantly less than with the other three groups (Figure 8). The results showed that PVA NFMs had little effect on fibroblast photomicrographs (Figures 7A and 7B). COS and FSCP treatments both showed good in vitro biocompatibility and supported proliferation of fibroblasts. FSCP combined with COS effectively enhanced the proliferation of fibroblasts (Figure 8). The MTT absorbance of FSCP, COS, and FSCP combined with COS treatments were similar to those of the negative control (fresh culture medium) and significantly different from those of the positive control (PVA) throughout the testing period. Chitin and chitin derivatives could accelerate the development of tensile strength in wounds by speeding fibroblastic synthesis of collagen in the first few days of wound healing. ${ }^{29}$ Carboxymethyl-chitosan, a water-soluble chitin derivative, was reported to stimulate the extracellular lysozyme activity of skin fibroblasts, and doubled the number of skin fibroblasts 


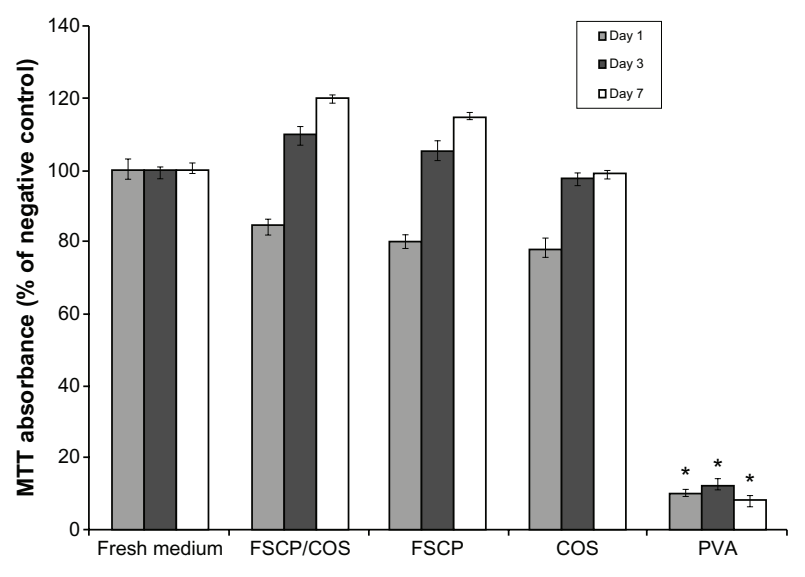

Figure 8 Fibroblast cell viability using the MTT assays. Fresh medium was used as a negative control and PVA as a positive control. The absorbance was normalized against the negative control at each time interval, which is taken as $100 \%$.

Note: $* P<0.05$ compared with the negative control.

Abbreviations: COS, chito-oligosaccharides; FSCP, fish scale collagen peptides; MTT, 3-(4,5-Dimethylthiazol-2-yl)-2,5-diphenyltetrazolium bromide; NFMs, nanofibrous membranes; PVA, polyvinyl alcohol.

while inhibiting the proliferation of keloid fibroblast cells. ${ }^{30}$ In this study COS could accelerate proliferation of normal human skin fibroblast cells. FSCP combined with COS in NFMs showed in vitro biocompatibility and may have the potential to be used as a functional biomaterial for wound dressing.

\section{Conclusion}

FSCP from fish scale collagen are superior to mammalian collagen, and are potential ingredients in wound dressing materials. To permit electrospinning of low MW FSCP and COS, PVA was added to enhance fiber formation. The antimicrobial activity of FSCP/COS NFMs is important for wound dressings. Studies using a representative Gram-positive bacterium, $S$. aureus, and a Gram-negative bacterium, E. coli, showed that the NFMs had a high bactericidal activity which was increased by COS content. Furthermore, the FSCP/COS NFMs had higher antimicrobial activity against $S$. aureus than against $E$. coli. The effects of FSCP/COS NFMs on bacterial adhesion suggested that bacteria adhere to the surfaces in different ways. In addition, COS with an average MW of $8 \mathrm{kDa}$ could accelerate normal human skin fibroblast proliferation. Thus, composite electrospun nanomembranes with FSCP/COS have good potential to be used as functional biomaterials for wound dressings.

\section{Acknowledgments}

This work was supported by the National Natural Science Foundation of China (No. 20976138 and No. 20576002), the Natural Science Foundation of Shanghai (No. 09ZR1434500), the Ministry of Agriculture of China (No. 2009ZX0800937B), and the China Postdoctoral Science Foundation (No. 20100470732). We thank Professor Joe M Regenstein for our English language copy editing, Dr Dongdong Lu for his technical support and kind help, and Ting Mo for her experimental help.

\section{Disclosure}

The authors declare that there are no conflicts of interest.

\section{References}

1. Kubik T, Bogunia KK, Sugisaka M. Nanotechnology on duty in medical applications. Curr Pharm Biotechnol. 2005;6:17-33.

2. Bognitzki M, Czado W, Frese T, et al. Nanostructured fiber via electrospinning. Adv Mater. 2001;13:70-72.

3. Reneker DH, Chun I. Nanometre diameter fibres of polymer, produced by electrospinning. Nanotechnology. 1996;7:216-223.

4. Fong H, Chun I, Reneker DH, Fong H, Chun I, Reneker DH. Beaded nanofibers formed during electrospinning. Polymer. 1999;40: 4585-4592.

5. Huang ZM, Zhang YZ, Kotaki M, et al. A review on polymer nanofibers by electrospinning and their applications in nanocomposites. Compos Sci Technol. 2003;63:2223-2253.

6. Yoshimoto H, Shin YM, Terai H, Vacanti JP. A biodegradable nanofiber scaffold by electrospinning and its potential for bone tissue engineering. Biomaterials. 2003;24:2077-2082.

7. Larsen G, Velarde-Ortiz R, Minchow K, Barrero A, Loscertales IG. A method for making inorganic and hybrid (organic/inorganic) fibers and vesicles with diameters in the submicrometer and micrometer range via sol-gel chemistry and electrically forced liquid jets. J Am Chem Soc. 2003;125:1154-1155.

8. Yang L, Fitié CFC, Werf KO, Bennink ML, Dijkstraa PJ, Feijen J. Mechanical properties of single electrospun collagen type I fibers. Biomaterials. 2008;29:955-962.

9. Rujitanaroj P, Pimpha N, Supaphol P. Wound-dressing materials with antibacterial activity from electrospun gelatin fiber mats containing silver nanoparticles. Polymer. 2008;49:4723-4732.

10. Herbert G, Reinhard S. Gelatine Handbook: Theory and Industrial Practice. Weinheim, Germany: Wiley; 2007.

11. Eckhart H, Werner R. Synthesis and investigation of collagen model peptides. Adv Polym Sci. 1982;43:143-203.

12. Tanaka M, Koyama YI, Nomura Y. Effects of collagen peptide ingestion on UV-B-induced skin damage. Biosci Biotech Bioch. 2009;73:930-932.

13. Kim SK, Kim YT, Byun HG, Nam KS, Joo DS, Shahidi F. Isolation and characterization of antioxidative peptides from gelatin hydrolysate of Alaska pollack skin. J Agric Food Chem. 2001;49:1984-1989.

14. Hassan CM, Peppas NA. Structure and applications of poly(vinyl alcohol) hydrogels produced by conventional crosslinking or by freezing/thawing methods. Adv Polym Sci. 2000;153:37-65.

15. Ong SY, Wu J, Moochhala SM, Tan MH, Lu J. Development of a chitosan-based wound dressing with improved hemostatic and antimicrobial properties. Biomaterials. 2008;29:4323-4332.

16. Liu SJ, Kau YC, Chou CY, Chen JK, Wu RC, Yehe WL. Electrospun PLGA/collagen nanofibrous membrane as early-stage wound dressing. J Membrane Sci. 2010;355:53-59.

17. Murakami K, Aoki H, Nakamura S, et al. Hydrogel blends of chitin/ chitosan, fucoidan and alginate as healing-impaired wound dressings. Biomaterials. 2010;31:83-90.

18. Min SK, Lee SC, Hong SD, Chung CP, Park WH, Min BM. The effect of a laminin-5-derived peptide coated onto chitin microfibers on re-epithelialization in early-stage wound healing. Biomaterials. 2010;17:4725-4730. 
19. Chen CZ, Cooper SL. Interactions between dendrimer biocides and bacterial membranes. Biomaterials. 2002;23:3359-3368.

20. Natthan C, Praneet O, Theerasak R, Tanasait N, Pitt S. Preparation and characterization of chitosan-hydroxybenzotriazole/polyvinyl alcohol blend nanofibers by the electrospinning technique. Carbohydr Polym. 2010;81:675-680.

21. Chen CZ, Mao JW, Wu QY. Preparation and conductivity of polyvinyl alcohol (PVA) films composited with molybdotungstovanadogermanic heteropoly acid. Mater Chem Phys. 2004;85:416-419.

22. Teo WE, Ramakrishna S. A review on electrospinning design and nanofibre assemblies. Nanotechnology. 2006;17:89-106.

23. Kim TG, Park TG. Biomimicking extracellular matrix: cell adhesive RGD peptide modified electrospun poly (D, L-lactic-co-glycolic acid) nanofiber mesh. Tissue Eng. 2006;12:221-233.

24. Proft T, Baker EN. Pili in Gram-negative and Gram-positive bacteria structure, assembly and their role in disease. Cell Mol Life Sci. 2009;66: 613-635.
25. Costerton JW. Introduction to biofilm. Int JAntimicrob Agents. 1999;11: 217-221.

26. Hall-Stoodley L, Costerton JW, Stoodley P. Bacterial biofilms: from the natural environment to infectious diseases. Nat Rev Microbiol. 2004;2: 95-108.

27. Boles BR, Horswill AR. Agr-mediated dispersal of Staphylococcus aureus biofilms. PLoS Pathog. 2008;4:1-13.

28. Jeon YJ, Park PJ, Kim SK. Antimicrobial effect of chitooligosaccharides produced by bioreactor. Carbohydr Polym. 2001;44:71-76.

29. Chung LY, Schmidt RJ, Hamlyn PF, Sagar BF, Andrews AM, Turner TD. Biocompatibility of potential wound management products: fungal mycelia as a source of chitin/chitosan and their effect on the proliferation of human F1000 fibroblasts in culture. J Biomed Mater Res. 1994;28:463-469.

30. Chen XG, Wang Z, Liu WS, Park HJ. The effect of carboxymethylchitosan on proliferation and collagen secretion of normal and keloid skin fibroblasts. Biomaterials. 2002;23:4609-4614.
International Journal of Nanomedicine

\section{Publish your work in this journal}

The International Journal of Nanomedicine is an international, peerreviewed journal focusing on the application of nanotechnology in diagnostics, therapeutics, and drug delivery systems throughout the biomedical field. This journal is indexed on PubMed Central, MedLine, CAS, SciSearch ${ }^{\circledR}$, Current Contents ${ }^{\circledR} /$ Clinical Medicine,

\section{Dovepress}

Journal Citation Reports/Science Edition, EMBase, Scopus and the Elsevier Bibliographic databases. The manuscript management system is completely online and includes a very quick and fair peer-review system, which is all easy to use. Visit http://www.dovepress.com/ testimonials.php to read real quotes from published authors. 